\title{
A relativistically broadened iron line from an Accreting Millisecond Pulsar
}

\author{
A.Papitto* $^{*}$ T.Di Salvo ${ }^{\dagger}$, A.D’Aì ${ }^{\dagger}$, R.Iaria ${ }^{\dagger}$, L.Burderi** $^{*}$, A.Riggio* $^{*}$ and \\ M.T.Menna \\ *INAF - Oss. Astr. di Cagliari, Poggio dei Pini, Strada 54, 09012 Capoterra (CA), Italy

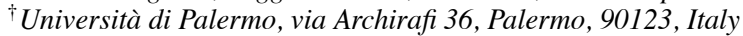 \\ ${ }^{* *}$ Università degli Studi di Cagliari, SP Monserrato-Sestu, KM 0.7, Monserrato, 09042 Italy \\ ${ }^{\ddagger}$ INAF Osservatorio Astronomico di Roma, via Frascati 33, Monteporzio Catone, 00040, Italy
}

\begin{abstract}
The capabilities of XMM-Newton have been fully exploited to detect a broadened iron $\mathrm{K} \alpha$ emission line from the $2.5 \mathrm{~ms}$ Accreting Millisecond Pulsar, SAX J1808.4-3658. The energy of the transition is compatible with fluorescence from neutral/lowly ionized iron. The observed large width (FWHM more than $1 \mathrm{keV}$ ) can be explained through Doppler and relativistic broadening from the inner rings of an accretion disc close to the NS. From a fit of the line shape with a diskline model we obtain an estimate of the inner disc radius of $18.0_{-5.6}^{+7.6} \mathrm{~km}$ for a $1.4 \mathrm{M}_{\odot}$ neutron star. The disc is therefore truncated inside the corotation radius (31 km for SAX J1808.4-3658), in agreement with the observation of coherent pulsations. From our estimate of the inner disc radius, we infer that the magnetic field of the neutron star is in the range $1-5 \times 10^{8} \mathrm{G}$.
\end{abstract}

Keywords: accretion, accretion disks - line: profiles - stars: pulsars: individual: SAX J1808.43658 - relativity - X-rays: binaries

PACS: $95.30 . \mathrm{Ky}, 97.10 . \mathrm{Gz}, 97.60 . \mathrm{Gb}, 97.60 . J d, 97.80 . J p$

\section{INTRODUCTION}

Much attention has been devoted in this conference to the most recent results obtained by $\mathrm{X}$-ray spectroscopy on the diagnostic possibilities offered by the broad emission features observed in the spectra of accreting collapsed objects, such as Active Galactic Nuclei or Galactic Low Mass X-ray Binaries (LMXB), hosting a Black Hole or a Neutron Star (NS). These features are generally interpreted as fluorescent emission due to the illumination of a geometrically thin, and optically thick, accretion disc by an external hard X-ray source. Their broadness is thus explained in terms of the relativistic Keplerian motion of the reflecting plasma in the accretion disc, under the gravitational influence of the compact object [1]. The shape of the line is determined by the size of the reflecting disc (that is, by its inner radius, $R_{\text {in }}$ and by its outer radius, $R_{\text {out }}$ ), by the index of the radial dependence of the line emissivity, and by the system inclination.

Here we consider the case of SAX J1808.4-3658 (J1808 in the following). This source was the first discovered [2], and thereafter most studied, Accreting Millisecond Pulsar (AMSP). An AMSP is a NS that accretes mass from a low mass companion star $(\lesssim 1$ $\mathrm{M}_{\odot}$ ) and shows coherent pulsations at a few milliseconds period. As the NS magnetic field is able to truncate the accretion disc at a radius $R_{i n}>R_{N S}$, where $R_{N S}$ is the NS radius, matter accretes only onto a small fraction of the NS surface at the magnetic caps. Pulsations at the spin period of the NS are observed if the magnetic axis is misaligned 
with respect to the spin axis.

J1808 is an X-ray transient source, like all the AMSP discovered so far. Since its discovery in 1996 [3], it shows a few weeks long outburst roughly every two years [see e.g. 4, and references therein]. Its broadband X-ray spectrum has been studied using RXTE data during its 1998 and 2002 episodes [5, 6, 7, 8]. Its 3.0-150 keV spectrum can be decomposed into a soft component originating from either the NS surface or the disc, and an energetically dominating hard component, interpreted as thermal Comptonization in a hot $\left(k T_{e}>35 \mathrm{keV}\right)$ medium [7]. Signatures of disc reflection, such as a $6.5 \mathrm{keV}$ iron emission line and a Compton hump at $\sim 30 \mathrm{keV}$ were also observed [7].

The observation of an emission line broadened by the relativistic motion in the accretion disc is invaluable for theories of accretion onto a pulsar, allowing a direct estimate of one of the key physical parameters, the inner disc radius. This radius represents the lever arm of the spin-up torque applied by the accreting matter on the NS. Motivated by this fact, we obtained a Target of Opportunity XMM-Newton observation of $\mathrm{J} 1808$ during its latest outburst. We were therefore able to fully exploit the high sensitivity and spectral resolution of XMM-Newton to constrain the $6.5 \mathrm{keV}$ line shape.

\section{THE XMM-NEWTON SPECTRUM OF J1808}

The 44 ks XMM-Newton observation we present here was performed during the latest outburst occurred in 2008 September/October [9]. The continuum spectrum observed by the EPIC-pn camera $(1.4-11 \mathrm{keV})$ is modelled using three continuum components, (i) the disc emission peaking at a temperature of $\mathrm{kT}_{\text {disc }}=0.24(1) \mathrm{keV}$; (ii) a 0.40 (2) $\mathrm{keV}$ black body coming from a region of radius $6.8(1) \mathrm{d}_{3.5} \mathrm{~km}$, where $d_{3.5}$ is the distance to the source in units of $3.5 \mathrm{kpc}$ [10]; (iii) an energetically dominating hard component, with a power law shape of index $\alpha=2.08(1)$ in the energy band in which XMM-Newton is sensitive. The presence of two soft thermal components to model the low-energy Xray spectrum has been also reported in the spectra of other similar sources $[11,12]$. The pulse shape and the decrease of the pulsed fraction with energy suggest that the blackbody and the hard component originate from the NS surface and from the accretion columns, respectively $[13,14]$. The hard component originates in the accretion columns, where the infalling matter is shocked by the interaction with the emergent radiation from below. The NS hotspots provide, in fact, the cool seed photons that downscatter the accreting hotter plasma through inverse Compton [see 11, and references therein].

Considering also the data taken by the Reflection Grating Spectrometers (RGS) in the 0.5-2.0 keV band, we estimate the equivalent hydrogen column of interstellar photoelectric absorption, $n H=0.214(3) \times 10^{22} \mathrm{~cm}^{-2}$. Also an edge at $E=0.871 \mathrm{keV}$, identified with OVIII absorption, is significantly detected.

A clear excess around $6.5 \mathrm{keV}$ is visible in EPIC-pn data (see left panel of Fig.1). If modeled with a Gaussian, a width of $\sigma=1.1(2) \mathrm{keV}$ is obtained. This large width can be explained with disc reflection of the hard continuum component. We thus consider a diskline model [1], which accounts for broadening by relativistic motion in the accretion disc near the NS. This model adequately describes the feature, indicating an inner disc radius in the range $6.0-12.4 \mathrm{R}_{g}$ (corresponding to $12.4-25.6 \mathrm{~km}$ for a 1.4 $\left.\mathrm{M}_{\odot} \mathrm{NS}\right)$. The energy of the transition is $6.43_{-0.09}^{+0.07} \mathrm{keV}$ and its equivalent width, $120(20)$ 

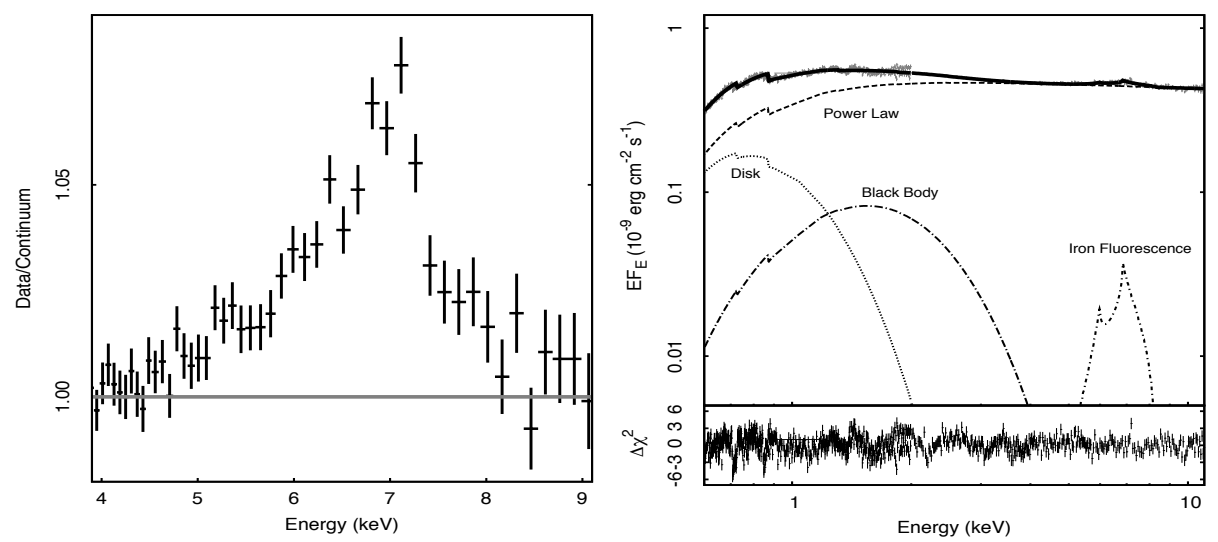

FIGURE 1. Ratio between data and continuum when the $6.5 \mathrm{keV}$ feature is not included in the model (left). 0.6-11 keV unfolded spectrum (right). The components of the best fitting model are also plotted. Spectral bins were rebinned for graphical purposes.

$\mathrm{eV}$. The index of the radial dependence of line emissivity, $\beta=-2.3(3)$, is consistent with with a compact illuminating source, and the inclination, $i \gtrsim 60^{\circ}$, is consistent with estimates made from completely independent arguments [15]. The improvement of the $\chi^{2}$ when using a diskline instead of a Gaussian profile $\left(\Delta \chi^{2}=13\right.$ for the addition of three parameters over 588 d.o.f.), together with the difficulty of explaining the observed width with alternative scenarios, supports the interpretation of the line profile in terms of disc reflection.

\section{DISCUSSION AND CONCLUSIONS}

The spectrum of J1808 observed by XMM-Newton is of unprecedented quality for what concerns AMSPs. It allows a firm detection of a broadened iron $\mathrm{K} \alpha$ feature at an energy compatible with neutral or mildly ionized iron. Despite a Gaussian symmetric profile cannot be excluded on a statistical basis, it is hard to find alternatives to relativistic motion of the reflecting plasma in the inner rings of the accretion disc, where the Gravitational field of the NS bends the space-time. Disc reflection is the only viable option to explain such a broad and low ionized feature, in a source whose spectrum is dominated by a Comptonized component originating from a hot, optically thin, medium. The line parameters we obtain are also compatible with those derived by a Suzaku observation performed during the same outburst [16].

The estimate we give for the inner disc radius is fundamental to test accretion theories. It allows to estimate where the NS magnetic field truncates the accretion disc. For an accreting pulsar, the inner disc radius $\mathrm{R}_{\text {in }}$ has to satisfy constraints implied by the presence of pulsations. It has to be larger than the NS radius, $R_{N S} \simeq 10 \mathrm{~km}$, and not much larger than the corotation radius, defined as the radius where the magnetosphere rotates at the same velocity of disc matter, which is assumed to be in Keplerian rotation. 
For $\mathrm{J} 1808$ the corotation radius is $R_{C}=\left(G M P^{2} / 4 \pi^{2}\right)^{1 / 3}=31 m_{1.4}^{1 / 3} \mathrm{~km}$, where $m_{1.4}$ is the NS mass in units of $1.4 \mathrm{M}_{\odot}$. If the condition $R_{\text {in }} \lesssim R_{C}$ were not met, the magnetosphere would have been spinning more rapidly than the disc at the inner disc radius, and accretion inhibited, or severely attenuated, by the centrifugal barrier [17]. The estimate we give for the inner disc radius from the iron line shape is $R_{i n}=18.0_{-5.6}^{+7.6} \mathrm{~m}_{1.4} \mathrm{~km}$. The lower bound overlaps with the NS radius, while the upper bound is perfectly consistent with the requirement $R_{\text {in }} \lesssim R_{C}$, for any reasonable NS mass. The value we measure can be also compared with theories that express $R_{\text {in }}$ in terms of the NS magnetic field strength and of the mass accretion rate [e.g. 18]. Estimating the mass accretion rate from the observed flux, the measured inner disc radius indicates a NS magnetic field in the range $1-5 \times 10^{8} \mathrm{G}$. These values are exactly in the plausible range $\left(10^{8}-10^{9} \mathrm{G}\right)$ for the AMSPs to be the progenitors of radio millisecond pulsars, according to the recycling scenario [19]. Moreover, they are compatible with estimates made from completely independent arguments [20].

The inner disc radius estimate could have also been useful if the $\mathrm{kHz}$ QPOs, already detected from this source [21], were observed in the power spectrum. Unfortunately this was not the case for this observation.

\section{ACKNOWLEDGMENTS}

We thank N.Schartel, who made this ToO observation possible. This work is supported by the Italian Space Agency, ASI-INAF I/088/06/0 contract for High Energy Astrophysics.

\section{REFERENCES}

1. A. C. Fabian, M. J. Rees, L. Stella, and N. E. White, MNRAS 238, 729-736 (1989).

2. R. Wijnands, and M. van der Klis, Nature 394, 344-346 (1998).

3. J. J. M. in 't Zand, J. Heise, J. M. Muller, et al., A\&A 331, L25-L28 (1998).

4. J. M. Hartman, A. Patruno, D. Chakrabarty, et al., ApJ 675, 1468-1486 (2008).

5. M. Gilfanov, M. Revnivtsev, R. Sunyaev, and E. Churazov, A\&A 338, L83-L86 (1998).

6. W. A. Heindl, and D. M. Smith, ApJ 506, L35-L38 (1998).

7. M. Gierliński, C. Done, and D. Barret, MNRAS 331, 141-153 (2002).

8. A. Ibragimov, and J. Poutanen, MNRAS 400, 492-508 (2009).

9. A. Papitto, T. Di Salvo, A. D’Aì, et al., A\&A 493, L39-L43 (2009).

10. D. K. Galloway, and A. Cumming, ApJ 652, 559-568 (2006).

11. M. Gierliński, and J. Poutanen, MNRAS 359, 1261-1276 (2005).

12. M. Falanga, J. M. Bonnet-Bidaud, J. Poutanen, et al., A\&A 436, 647-652 (2005).

13. J. Poutanen, and M. Gierliński, MNRAS 343, 1301-1311 (2003).

14. A. Patruno, N. Rea, D. Altamirano, et al., MNRAS 396, L51-L55 (2009).

15. C. J. Deloye, C. O. Heinke, R. E. Taam, and P. G. Jonker, MNRAS 391, 1619-1628 (2008).

16. E. M. Cackett, D. Altamirano, A. Patruno, et al., ApJ 694, L21-L25 (2009).

17. A. F. Illarionov, and R. A. Sunyaev, $A \& A$ 39, 185-+ (1975).

18. P. Ghosh, and F. K. Lamb, ApJ 232, 259-276 (1979).

19. D. Bhattacharya, and E. P. J. van den Heuvel, Phys.Rep. 203, 1-124 (1991).

20. T. Di Salvo, and L. Burderi, A\&A 397, 723-727 (2003).

21. D. Chakrabarty, E. H. Morgan, M. P. Muno, et al., Nature 424, 42-44 (2003). 